\title{
Différence de capacité digestive et d'efficacité alimentaire entre des taurillons culards de race Blanc-Bleu-Belge issus de 3 taureaux
}

\author{
AG Deswysen, HA Falys, JM Embrechts, EH Amouche \\ Université catholique Louvain, Fac Sci Agr, unité de génétique, place Croix-du-Sud, 2 (bte 14), \\ 1348 Louvain-la-Neuve, Belgique
}

L'objectif du présent travail est d'étudier les possibles différences génétiques de capacité de digestion de la matière sèche (dMS) et d'efficacité alimentaire au sein de la race Blanc-Bleu-Belge (BBB) cularde.

Trois taureaux furent choisis sur la base de leur croissance journalière entre 7 et 13 mois : Sénior $(1,38 \mathrm{~kg} / \mathrm{j})$, Hamac $(1,60 \mathrm{~kg} / \mathrm{j})$ et Infernal $(1,83 \mathrm{~kg} / \mathrm{j})$. Chez 7 fils de Sénior, 11 fils de Hamac et 16 fils d'Infernal, le gain de poids vif et la consommation d'aliments furent mesurés entre 8 et 13 mois et la dMS fut mesurée à l'âge de $325 \pm 23 \mathrm{j}$ (poids vif : $412 \pm 41 \mathrm{~kg}$ ). Ces taurillons requrent en libre choix du concentré $(16,5 \%$ MAT, $39,7 \%$ NDF) et du foin (6,0 MAT, 68,2 NDF) dans des auges individuelles équipées de portillons Calan. Ils furent logés par groupe de 5, sur litière de paille, et sur sciure de bois lors de la mesure de la dMS (pendant $42 \mathrm{j}$ ). Au cours des 21 derniers $\mathrm{j}$, un échantillon de matières fécales fut prélevé dans le rectum, 2 fois par semaine. La dMS fut déterminée en utilisant l'ADF indigestible à la cellulase comme marqueur interne $\left(168^{\circ} \mathrm{h}\right.$ d'incubation ; Jochemsen et Testering, 1990). Les taurillons furent abattus à l'âge de 13 mois. Le temps de séjour moyen (TSM) des digesta dans le tractus digestif proximal (TDP : 4 compartiments gastriques) fut estimé par le rapport du poids de digesta présent dans le TDP $(\mathrm{kg}$ MS)/consommation journalière de concentré avant l'abattage ( $\mathrm{kg} \mathrm{MS/j})$.

L'effet patemel fut significatif $(P<0,10)$ pour l'ingestion volontaire, la proportion de foin dans la ration, la dMS et le TSM (tableau I). La dMS fut positivement corrélée avec la proportion de foin dans la ration $(n=34 ; r=+0,41 ; P=0,02)$ et le $\operatorname{TSM}(r=+0,39 ; P=0,04)$, mais non avec l'ingestion volontaire. Le TSM fut négativement corrélé avec l'indice de consommation $(r=-0,48$; $P=0,01$ ). La meilleure capacité de digestion, spécialement des descendants de Sénior, semble être partiellement liée au choix d'une ration à degré de structure plus élevé permettant un TSM plus long. et probablement une meilleure activité mastica. toire et une production salivaire plus importante.

Recherche réalisée avec la collaboration financière du ministère de la région Wallonne-Agriculture.

Jochemsen J, Testering J (1990) IVVO inteme mededeling, Lelystad, Holland

Tableau I. Ingestion volontaire, proportion de foin dans la ration et digestibilité de la MS à l'âge de 11 mois, temps de séjour moyen (TSM) des digesta dans le tractus digestif proximal (TDP) à 13 mois, indice de consommation et croît journalier entre 8 et 13 mois des taurillons issus de 3 taureaux.

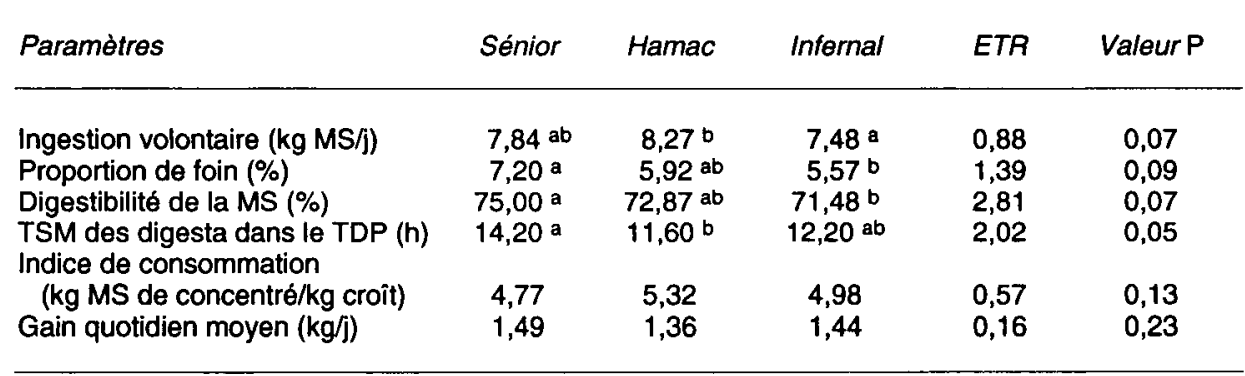

Les moyennes sur une même ligne sans une lettre en commun diffèrent $(P<0,05)$. 